\title{
EVALUATION OF WOOD SURFACE MODIFICATION USING HOT MELTING PLASTIC DROPLETS FOR CONTACT ANGLE MEASUREMENTS
}

\author{
POURIA REZAEE NIARAKI \& ANDREAS KRAUSE \\ Centre of Wood Science University, Germany
}

\begin{abstract}
There are several methods to measure the contact angle between the solid surface and liquid phase. One of the most applicable ones is known as the sessile drop method, on which a liquid droplet is deposited on a solid surface to measure the contact angle by a high-resolution camera. In this paper, molten thermoplastic droplets are placed directly on the surface of modified wood in order to assess the compatibility between hydrophobic thermoplastics and hydrophilic wood surfaces. For this purpose, two groups of spruce veneers are treated with acetic acid anhydride (AA) or maleic acid anhydrides (MA), respectively. Droplets from high density polyethylene (HDPE) are deposited on the wood surfaces at isothermal conditions. The results show that thermoplastic droplets exhibited higher contact angles at AA treated surfaces than at MA treated ones. This suggests that MA reduces the polarity of the wood surface compared to AA, which has an increased compatibility with wood surface.

Keywords: wood surface, high density of polyethylene (HDPE), contact angle, acetylation.
\end{abstract}

\section{INTRODUCTION}

There is a great interest to reduce the usage of non-renewable materials by using biocomposite materials. Natural fiber-reinforced composites resulting from the compounding of lignocellulosic material with thermoplastic polymer matrices have been developed to take advantage of the large quantity, annual renewability, low cost, light weight, excellent and competitive specific strength, and environmental friendliness of such material [1]. However, there are still some serious obstacles to achieve such purposes. Wood plastic composite is a compound produced using wood powder or wood fibers or other lignocellulosic products as reinforcing material to improve mechanical and physical properties of the plastics. However, the incompatibility between hydrophilic wood phase and hydrophobic plastic causes a critical barrier ahead to combine these heterogeneous materials and obtain a material with acceptable physical and mechanical properties. There were lots of attempts to improve the interaction between these two compartments. Either by changing on plastic matrix or carrying out a chemical treatment of wood fibers in order to optimize the interfacial adhesion. However, there were always critical challenges for scientists to select the correct experimental device to evaluate the effect of such chemical change on wood composites. For interfacial adhesion between different substrates is difficult to measure the probable reactions occurred on their contact surfaces. Generally, adhesion is pronounced when two surfaces are held together by interfacial forces [2]. Law and Zhao [3] announced that wettability and adhesion are the most common properties of a surface which can be characterized both by contact angle measurements. The contact angle is the angle when a liquid droplet wets a solid surface and the angle between the liquid and solid surface is measured to determine the adhesion property of the solid. This experiment is usually carried out by several different liquids e.g. water, glycol and diiodomethane. Theoretically each of them has been selected to represent the features of a polar or non-polar liquid but in practice there is a discrepancy between the physical and chemical properties of such a liquid and the molten plastic, which is compounded together with wood. Up to now, there is no published investigation using melted 
plastic droplet as the applicable liquid to measure the contact angle directly on wood surfaces. This paper shows that this technique gives different results for different wood surface chemistry. We investigated the impact of acetylation with acetic acid anhydride and maleic acid anhydride treatment on wood surfaces by measuring the contact angle of a molten droplet of high density of polyethylene (HDPE).

\section{MATERIAL AND METHODS}

Veneers of Norway spruce (picea abies) were cut to following dimensions $117 \times 20 \times 0.6 \mathrm{~mm}$. The veneers were divided into three groups: non-treated (REF), treated with acetic acid anhydrides (AA) and treated samples with maleic acid anhydrides (MA) respectively. The veneers were extracted by soxhlet technique using mixture of ethanol-water (4:1) for 4 hours and were dried in an oven at $103 \mathrm{C}$ for approximately $24 \mathrm{~h}$. For AA treatment, wood veneers were heated with a 1:1-part mixture of acetic anhydrides and pyridine solvent over $1 \mathrm{~h}$ at $90^{\circ} \mathrm{C}$. For maleic anhydride treatment, the samples were also heated under the same condition with a 1:2-part mixture of maleic anhydride and dimethylformamide (DMF) solvent, respectively. At the end of process, all treated veneers were dried at $103^{\circ} \mathrm{C}$ for about $24 \mathrm{~h}$. The quality of modification was evaluated by weight percent gain (WPG) All treated samples were acetylated to an approximate WPG of $20 \%$ in order to achieve the equal condition for experimental calculations. FTIR (Fourier transform infrared spectroscopy) spectrometer used infrared light to scan test samples and detect functional groups on wood surface.

The contact angle measurements were carried out using high temperature dosing system of drop shape analyzer (DSA 100, Krüss, Hamburg, Germany). HDPE (cc3054) provided from SABIC was heated at $190^{\circ} \mathrm{C}$ and - under isothermal conditions - a melted plastic droplet was deposited on the wood veneer surface. The advancing contact angle $\Theta a$ was measured for each droplet after 10s from the moment the droplet was deposit on the wood surface in order to stabilize the droplet position. In order to avoid distortion of drop shape as the result of Gravity or laboratory conditions, a drop size of $3 \mu \mathrm{m}$ was used for each measurement and the tangent method was applied to measure the contact angles on the wood surfaces.

\section{RESULTS AND DISCUSSIONS}

The principal aim of acetylation treatment is to substitute hydroxyl groups on wood surface by carbonyl groups of acetyls to improve dimensional stability and interfacial compatibility in wood reinforced composites [4], [5]. Fig. 1 illustrates the IR band results from non-treated and treated samples. According to Infrared Spectroscopy Absorption Table, the region 1600$1800 \mathrm{~cm}^{-1}$ is allocated for bonding carbonyl groups on the wood surfaces [6]. As it is shown, treated samples with AA showed higher peak than treated samples with maleic anhydride. However, for the MA, there are two peaks of carbonyl bonds $\left(1642 \mathrm{~cm}^{-1}, 1725 \mathrm{~cm}^{-1}\right)$ derived from the main difference in the chemical structure of cyclic maleic anhydride and linear acetic anhydride. When the treatment is carried out by acetic anhydride there are always more carboxylic compounds at disposal but due to the unstable structure of linear acetic anhydride and its incompatibility with wood chemical morphology, they are likely to form an unconjugated structure. Therefore, FTIR peak showed $1738 \mathrm{~cm}-1$ wave number by AA treatment which is normally attributed to unconjugated carbonyl groups on the wood surface [7]. On the contrary, when maleic anhydride reacts with wood components, there is always one more carbonyl group per reaction $(1642 \mathrm{~cm}-1)$ which is called as conjugated carbonyl group and forms much more stable structure than acetic groups (Figs 1 and 2) [6]. 


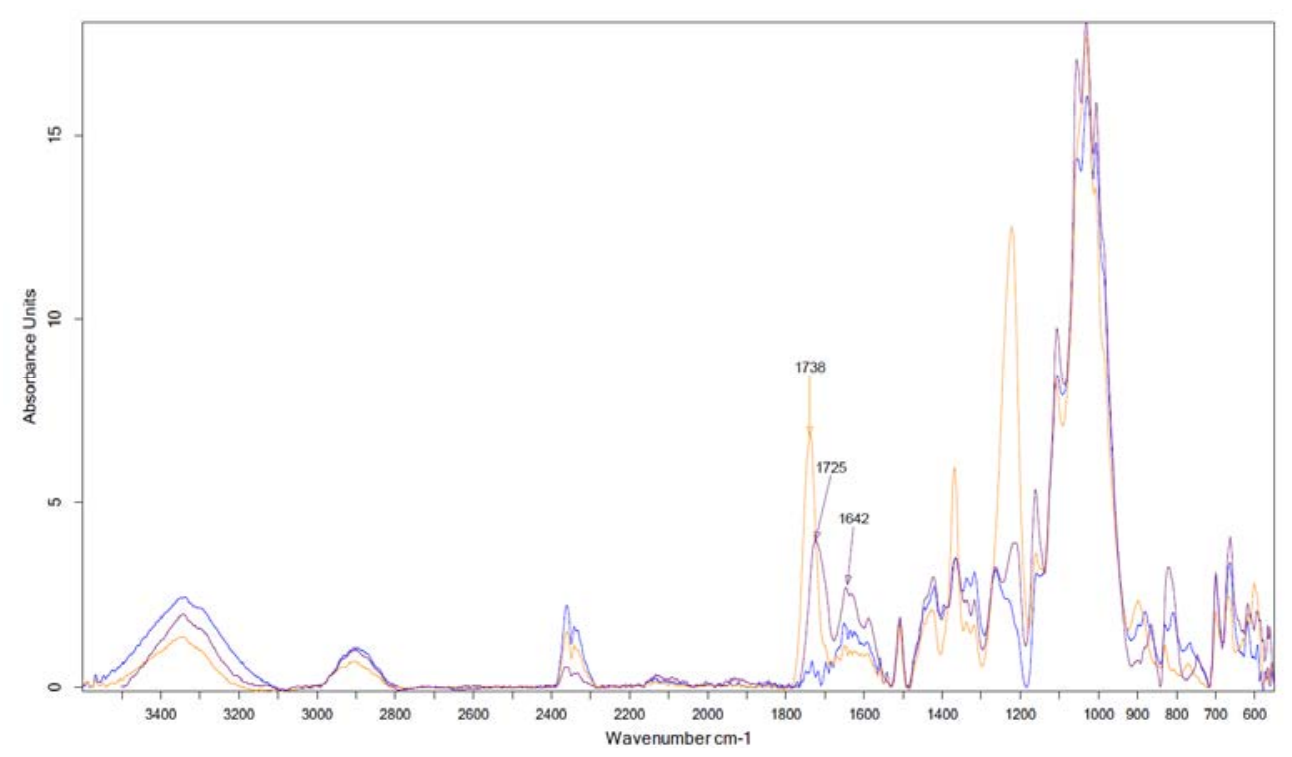

Figure 1: FTIR spectra of REF (yellow color), MA (blue color) and AA (pink color) treated samples.

One purpose of acetylation treatment is to decrease the surface free energy of wood and close its properties to the hydrophobic plastic phase in the composite [8]. However, there were always disagreements among scholars on whether in fact this treatment has an effective impact on wood adhesion properties. Generally, in chemical science, wetting is known as the liquid ability to maintain contact with solid surface. The more a drop of liquid spreads on solid surface, the more wetting values for solid can be assessed consequently. Fig. 3 shows the result from the contact angle measurements between HDPE droplet and wood veneer. As it is indicated AA treated samples had partially the highest contact angles compared to both REF and MA ones. This is unlike general believes on which there is expected to have more hydrophobic surface property after acetylation treatment and lower contact angle should be achieved between hydrophobic HDPE droplet and wood veneers consequently. On the contrary, the current observation shows that AA makes the wood surface more insusceptible to react with unpolar plastic droplet than REF samples. This is in accordance with Liu, Feipeng et al. [9] founding that acetylation increased the surface free energy of wood specimens. They announced that by the replacement of hydroxyl groups in wood structure with acetyl groups, fiber surfaces are dominated by the ester configuration which have more polar characteristic than hydroxyls groups. On the other hand, MA treatment showed the lowest contact angle. This is supported by its wide application of MA grafted on PE or PP as a coupling agent for wood plastic composites. This is also in accordance with Rowell and Clemons [10], where they concluded that reaction of MA with wood made the wood characteristic similar to thermoplastic phase and it does thermoform the wood fiber into a high-density composite under pressure. 
<smiles>CC(=O)OC(C)OC(C)=O</smiles>

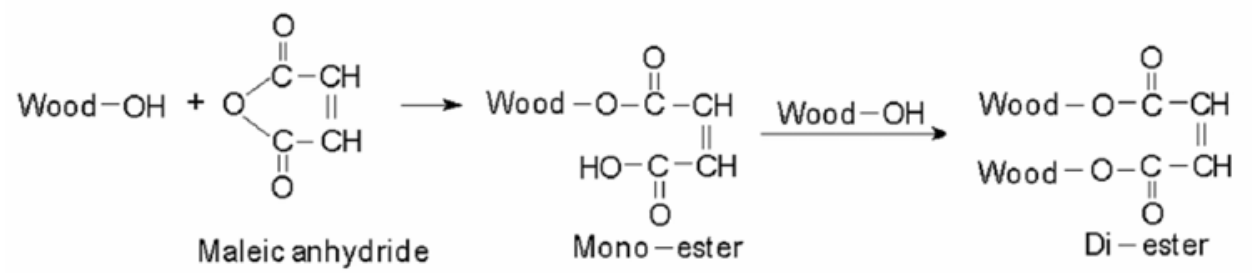

Figure 2: (a) Chemical reaction of AA with wood; (b) Chemical reaction of MA with wood.

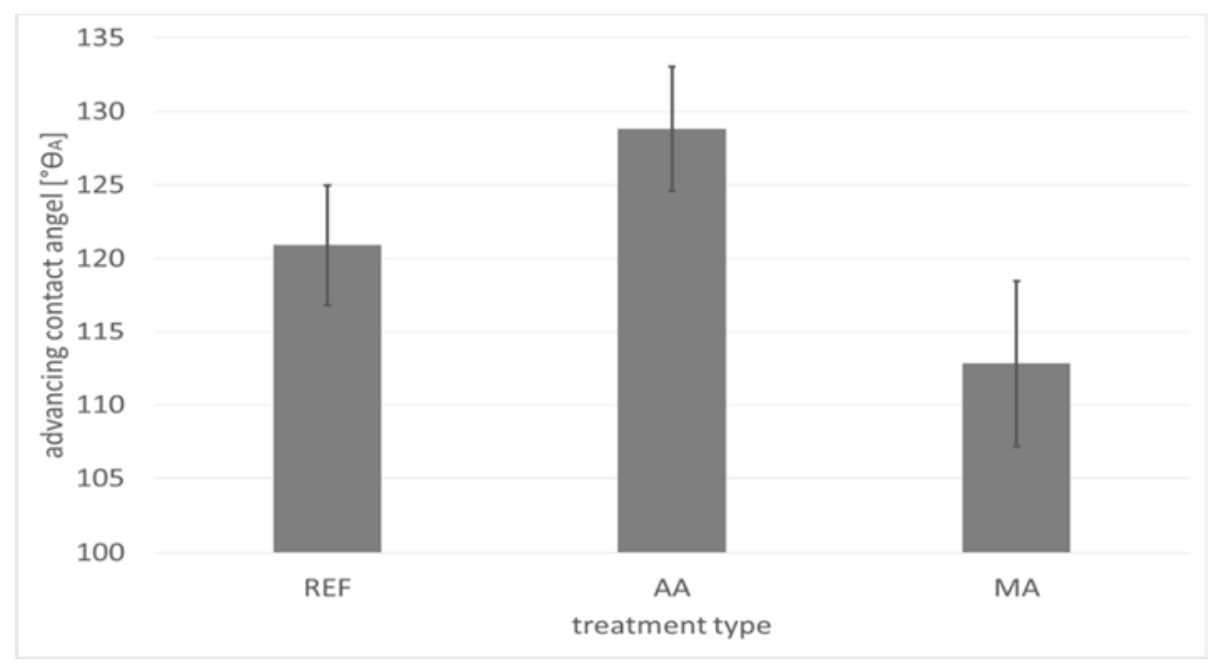

Figure 3: The contact angle measurements between wood surfaces and HDPE hot droplet.

There are two main aspects on which the function of both acetic and maleic anhydrides can be separated from each other. On one hand, the difference between the chemical structures of these two anhydrides are of main target. Generally, the application of acid as a catalyst is avoided. Since it can also destroy the polysaccharides components of wood. Furthermore, the presence of acid could dilute the active anhydride reagents, which would solely decrease the rate of reaction on wood. On the other hand, there is a critical difference between the types of structures on which these two chemicals are usually formed. The usages of linear anhydrides like acetic anhydride on wood are totally restricted. Since longer chain 
anhydrides have less potential to bond with cellulose components of wood structure [7]. Furthermore, surface interactions and forms of final chemical reactions between wood and chemical substance are of more importance than the natural ability of substance to increase hydrophilicity or hydrophobicity of wood surface. There are some new evidences showing that MA treatment does not hydrophobize wood fibers but it increases the gluing ability of wood in reaction to other chemicals [11].

\section{CONCLUSIONS}

Contact angle measurements were carried out using hot melting plastic droplet on nontreated, acetic anhydride and maleic anhydride treated wood samples. To diagnose the chemical differences, FTIR spectrometer analyzed ester groups on wood surface. Based on experimental observations, following statements were concluded:

- Treated samples with MA improved wettability on wood surface while AA modification had some negative impact on this factor. This is probably because of the general compatibility/incompatibility between the chemical structures of cyclic/linear anhydrides and wood respectively.

- Regardless of intensity of carbonyl presence in wood treated samples, it is of more importance to obtain interfacial bonding between ester groups and wood cells.

- Introducing the new technique of contact angle measurement to diagnose any critical alteration of wood surface can play a determining role in the future path of this field of research.

\section{REFERENCES}

[1] Yao, F., Wu, Q., Lei, Y \& Xu, Y., Rice straw fiber-reinforced high-density polyethylene composite: effect of fiber type and loading. Industrial crops and products, 28(1), pp. 63-72, 2008

[2] Gilbert Gedeon, P.E., Adhesive bonding of wood materials.

[3] Law, K.Y. \& Zhao, H., Surface wetting: characterization, contact angle, and fundamentals, Springer, 2015.

[4] Stefke, B., Windeisen, E., Schwanninger, M. \& Hinterstoisser, B., Determination of the weight percentage gain and of the acetyl group content of acetylated wood by means of different infrared spectroscopic methods. Analytical chemistry, 80(4), pp. 1272-1279, 2008.

[5] Gurunathan, T., Mohanty, S. \& Nayak, S.K., A review of the recent developments in biocomposites based on natural fibres and their application perspectives. Composites Part A: Applied Science and Manufacturing, 77, pp. 1-25, 2015.

[6] Naumann, A., Peddireddi, S., Kües, U. \& Polle, A., Fourier Transform Infrared Microscopy in Wood Analysis. Wood production, wood technology, and biotechnological impacts, 179, 2007.

[7] Jahan, M.S., Chowdhury, D.N., Islam, M.K. \& Moeiz, S.I., Characterization of lignin isolated from some nonwood available in Bangladesh. Bioresource technology, 98(2), pp. 465-469, 2007.

[8] Hill, C.A., Wood modification: chemical, thermal and other processes, John Wiley \& Sons, 2007.

[9] Liu, Feipeng, P. et al., "Characterization of the interface between cellulosic fibers and a thermoplastic matrix." Composite Interfaces, 2.6, pp. 419-432, 1994.

[10] Rowell, R.M. \& Clemons, C.M., Chemical modification of wood fiber for thermoplasticity, compatibilization with plastics, and dimensional stability. In 
36 Materials and Contact Characterisation VIII

Proceedings of Washington State University International Particleboard/Composite Materials Symposium (USA), 1992.

[11] Hundhausen, U., Kloeser, L. \& Mai, C., Usability of maleic anhydride as wood modification agent for the production of medium density fibreboards (MDF). European Journal of Wood and Wood Products, 73(3), pp. 283-288, 2015. 